\title{
A dermatology health services study of nursing homes in Singapore
}

\author{
Adeline Mei Yen Yong ${ }^{1}$, MRCP, An Jian Leung ${ }^{2}$, MBBs, Nisha Suyien Chandran ${ }^{1}$, MRCP
}

\section{INTRODUCTION}

The global healthcare landscape is shifting from specialised tertiary care towards comprehensive primary and preventive care. Like many developed nations, Singapore is facing an ageing crisis. The proportion of residents aged 65 years and above has increased from $8.7 \%$ in 2008 to $13.7 \%$ in $2018 .^{(1)}$ The greater demand for elderly healthcare necessitates step-down and longer-term care facilities, including community rehabilitation, nursing homes and chronic sick units.

Furthermore, although much is known about the burden of chronic cardiovascular diseases in our population, data is scant on the burden of skin conditions. A randomised controlled trial in 2008 evaluating nurse clinic versus home delivery of community leg ulcer care showed that it was the organisation and not the setting of care that influenced healing rates. ${ }^{(2)}$ Key factors included the delivery of evidence-based recommendations with care provided by a trained nursing team.

The present study aimed to assess the prevalence and range of skin conditions in various step-down facilities including nursing homes, community hospitals and chronic sick units in Singapore. We also aimed to review the available medial and nursing resources within the step-down facilities and assess the patterns of utilisation of external medical care. This information could allow identification of potential areas for improvement and implementation of protocols for optimisation of dermatological care.

\section{METHODS}

We performed a prospective multi-centre descriptive study on step-down care services among residents in step-down facilities in Singapore. This study was carried out from January to June 2017. Institutional review board approval was obtained.

Institutions in the West catchment area were identified in view of proximity to our centre, and six nursing homes and one community hospital were selected. These comprised both privately funded hospitals as well as those managed by volunteer welfare organisations. A total of three nursing homes consented to participate. Patient inclusion criteria were (a) being a resident of the respective residential care facility; and (b) having a documented skin condition before or during the study period. Residents with a diagnosis of pressure sores were excluded.

Our study was twofold. First, a questionnaire was sent to various institution heads at the start of the study to assess the epidemiology of residents as well as current nursing and doctor manpower profiles. Next, monthly reviews of patients' medical records were performed by the authors (Yong AMY and Leung AJ). The types of skin conditions among patients and the follow-up location were assessed.

The operational workflow is summarised in Fig. 1. Upon documentation of a skin issue in the case notes, the resident's records were reviewed to ascertain if a dermatological diagnosis was made prior to data collection or whether the ongoing skin issue had yet to be evaluated by a doctor (i.e. new skin issue). For residents whose dermatological diagnoses were made prior to data collection, previously prescribed medication was continued. Worsening conditions were addressed by an in-house general practitioner (GP) or external dermatology specialists. Conversely, residents with new-onset skin issues and those who had ongoing skin issues that had not yet been evaluated by a doctor received empirical treatment with creams issued by nurses. These included emollients, topical anti-fungals, and combination steroid and anti-microbial creams. Depending on whether the skin issues persisted, an in-house GP review or external specialist dermatology consultation was arranged.

\section{RESULTS}

The demographics of the residents are shown in Table I. 489 residents from three nursing homes were screened for skin conditions. Their mean age was 81.3 years. The average number of nursing staff for each home was 58.7, with a patient-to-nurse ratio of $2.78: 1$. The majority were nursing aides $(63.6 \%$ of total nursing staff). Only one nurse educator was available throughout the study. The average number of in-house doctors was one per nursing home. There were no volunteer doctors. An average of 2.3 nearby GP clinics for each home were available for referrals.

During the six-month study period, $34(7.0 \%)$ residents had skin issues, with a male-female ratio of 1:1.27 (Fig. 2). All patients with skin conditions were Chinese. 30 residents had skin issues at the start of the study prior to data collection, of which 19 diagnoses were made by a GP and 11 at an acute hospital. Another four residents developed skin issues during the study period. In terms of admission diagnoses, the majority (44.1\%) of the 34 residents with skin issues were admitted for chronic medical illnesses, 38.2\% for dementia and $29.4 \%$ for falls (Fig. 3). Chronic medical illnesses included heart, liver or renal failure; hypertension; hyperlipidaemia; and diabetes mellitus. $8.8 \%$ of

${ }^{1}$ Division of Dermatology, University Medicine Cluster, National University Hospital, ${ }^{2}$ NUS Yong Loo Lin School of Medicine, National University of Singapore, Singapore Correspondence: Dr Adeline Yong Mei Yen, Division of Dermatology, University Medicine Cluster, National University Hospital, 5 Lower Kent Ridge Road, Singapore 119074. adeline_yong@nuhs.edu.sg 


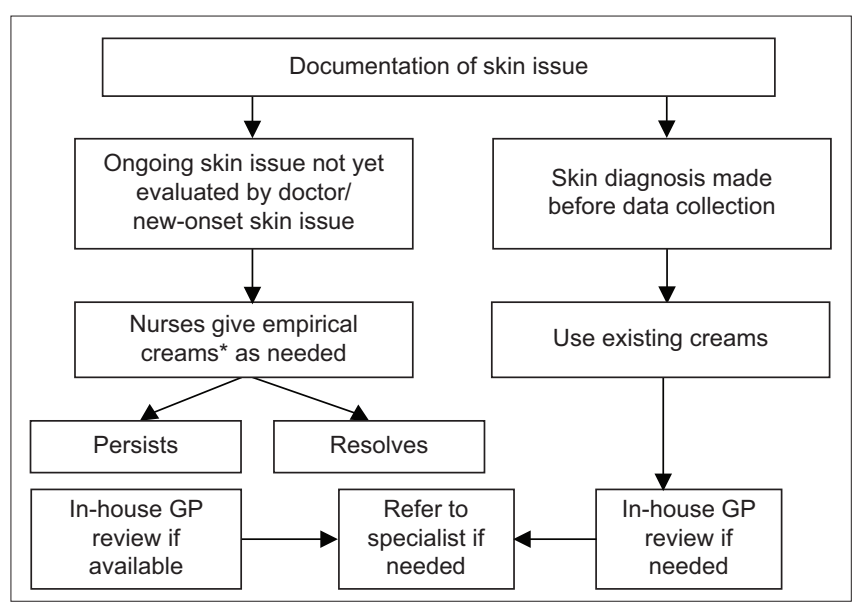

Fig. 1 Flowchart shows operational workflow by nurses upon encountering a documented skin issue. *Empirical creams such as emollients, topical anti-fungals or a combination of topical steroids with topical anti-fungal and antibiotic agents. GP: general practitioner

Table I. Profile of nursing home residents from survey $(n=489)$.

\begin{tabular}{|ll|}
\hline Characteristic & No. (\%) \\
\hline Gender & \\
\hline Male & $200(40.9)$ \\
\hline Female & $289(59.1)$ \\
\hline Ethnicity & \\
\hline Chinese & $452(92.4)$ \\
\hline Malay & $14(2.9)$ \\
\hline Indian & $9(1.8)$ \\
\hline Caucasian & $1(0.2)$ \\
\hline Others & $13(2.7)$ \\
\hline
\end{tabular}

the patients had an underlying malignancy and $2.9 \%$ had an underlying psychiatric disorder. The mean age at admission was 83.4 years. The most prevalent skin conditions were eczema $(70.6 \%)$, followed by tinea infections (35.3\%), cellulitis (14.7\%), scabies (11.8\%) and bullous pemphigoid (8.8\%) (Fig. 4). Occupancy rates at the nursing homes were stable throughout the study period, only differing by a maximum margin of 5\% every month. Hence, the cumulative incidence rate for patients with skin issues was calculated to be $7.0 \%$.

Across the study period, 24 residents among the 34 in the cohort were reviewed a total of 35 times, with an average of 1.48 times per patient. Of these reviews, $60.0 \%$ were by an in-house GP or at a nearby polyclinic/GP, $20.0 \%$ were by dermatologists at private or specialist centres, and $20.0 \%$ were by dermatologists or other physicians at acute hospitals. Age and number of comorbidities were not significantly associated with the number of reviews ( $p=0.974$ and $p=0.159$, respectively). The average duration from onset of skin issue to review was 2.03 months. Diagnoses of the reviewed patients included eczema (54.2\%), tinea infection $(20.0 \%)$, cellulitis/scabies infection $(11.4 \%)$ and bullous pemphigoid $(8.6 \%)$. Ten patients were not reviewed during the sixmonth study period due to resolution of skin conditions or family members declining further reviews on the patient's behalf. All patients who were not reviewed had a pre-existing skin diagnosis made prior to data collection (seven diagnoses of eczema, four diagnoses of tinea infections and one basal cell carcinoma).

\section{DISCUSSION}

Epidemiological studies suggest that dermatological diseases are highly prevalent in the elderly, and that the global burden of skin conditions increases with age. ${ }^{(3-5)}$ However, the majority of the data was obtained in acute hospital settings. Limited information exists in the context of community-based facilities. ${ }^{(6)}$ This is despite a global increase in the number of multimorbid residents in institutional long-term care. (7) $^{\text {(n) }}$

Skin disease is associated with a significant impairment of one's quality of life. ${ }^{(8)}$ Geriatric patients with skin conditions have a predilection for mental and behavioural disorders, especially depression. ${ }^{(9)}$ In turn, the co-existence of immobility, cognitive impairment and organisational factors limit available specialised dermatological care for such residents.

Our data demonstrates the significance of skin disease in nursing homes, with a prevalence of $7.0 \%$ of residents with reported skin issues. In contrast, a recent German prevalence study showed that nearly every resident in institutional care is affected by at least one dermatological disease. ${ }^{(10)}$ This discrepancy can be explained by the presence of a head-to-toe skin examination by board-certified dermatologists in the German study, versus a review of medical records on documented skin issues in our study. The existing literature indicates that tinea infections, xerosis cutis, seborrhoeic keratosis and androgenetic alopecia are among the most common skin conditions in nursing homes. ${ }^{(11-13)}$ This is similar to our study, where tinea infections including tinea ungiuum and tinea pedis were common. The disease burden of skin conditions in nursing homes is comparable to the prevalence of diabetes mellitus in Singapore. Based on a Ministry of Health consensus, among those aged 18-69 years in 2017, the prevalence of diabetes mellitus stands at $8.6 \%$, hypertension at $21.5 \%$ and hyperlipidaemia at $33.6 \%$. ${ }^{(14)}$

Furthermore, age and number of comorbidities were not significantly associated with number of reviews ( $p=0.974$ and $p=0.159$, respectively), which may suggest that care is not stratified to age or background comorbidities. Expectedly, persons with multiple chronic conditions are particularly vulnerable to suboptimal care. ${ }^{(15)}$ This is because they tend to use services more frequently and in greater variety, which makes coordination of care more difficult. As such, stratification of care to background comorbidities and age, in which patients with more advanced age and more comorbidities have more frequent clinical reviews and resources allocated, may be beneficial.

Overall, $60 \%$ of patients were reviewed by in-house GPs and nearby polyclinics; hence, the majority of residents can be considered to have been reviewed in-house. This, in our opinion, is the ideal state, as there is less movement of patients and reflects a decreased need for further resources in transporting patients from one facility to another. Review on-site can be performed by dermatologists, the primary care physician or nurses, highlighting the importance of education to increase the knowledge and management of skin diseases by all the healthcare providers at these step-down facilities. Our data showed a patient-to-nurse ratio of 


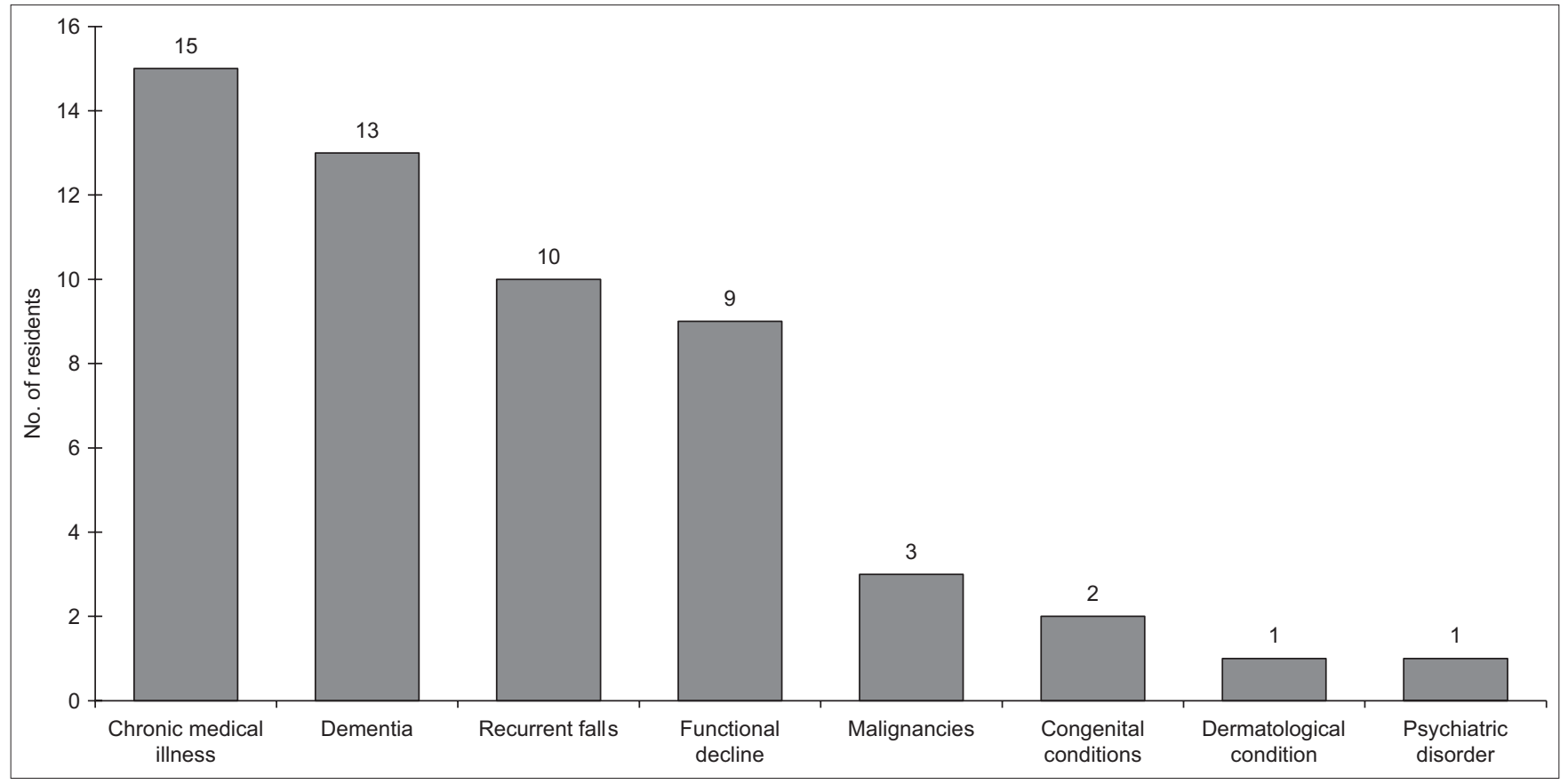

Fig. 2 Chart shows admission diagnoses of patients with skin conditions.

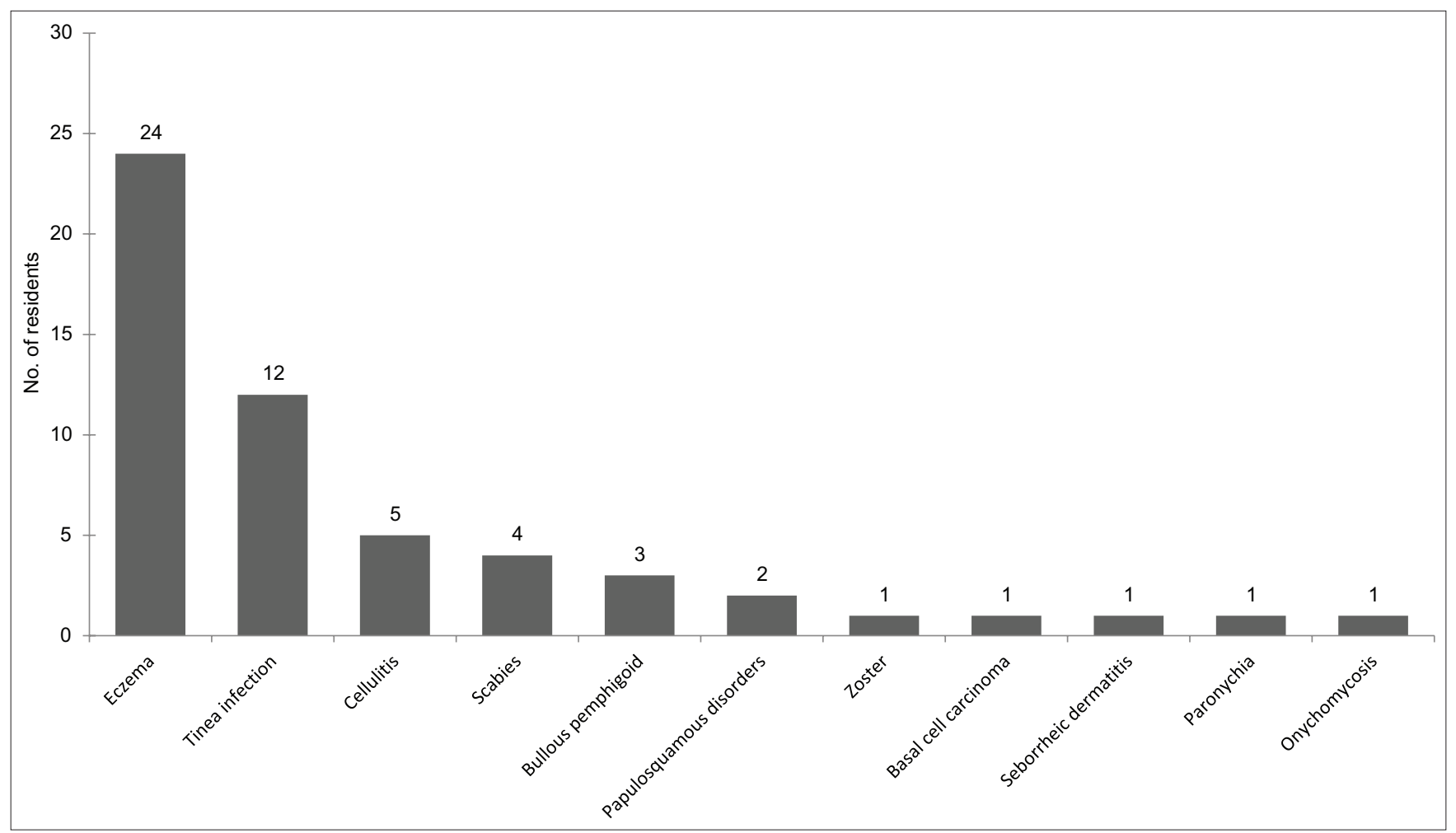

Fig. 3 Chart shows dermatological diagnoses of patients with skin conditions.

2.78:1. The Ministry of Health subvention and staff ratios are based on the assessment status of the physical, psychological, emotional and social needs of nursing home residents. ${ }^{(14)}$ This is divided into four care categories (I-IV) based on needs. For Category IV residents who are bedridden and require total assistance and supervision for every aspect of activities of daily living (ADLs), the optimal staff-to-patient ratio has been denoted as 1:2. For Category III residents who are wheelchair bound and/or suffering from dementia or need help in ADLs and supervision most of the time, the optimal ratio is 1:4. For Category II residents who are semi-ambulant and/or mildly senile and require some physical assistance and supervision in ADLs, the optimal care staff-to-patient ratio is 1:8. Category I patients who are physically and mentally independent and may only need minimal assistance in ADLs (if any) are not intended for nursing home facilities. As nurses are often the first-line healthcare providers to the residents, improving their dermatological knowledge would be of direct benefit to patient care. In this regard, the training of a nurse educator with 
a special interest in dermatology would be an effective means of cascading knowledge to nursing staff across step-down facilities. The employment of teledermatology between primary care doctors at step-down facilities and remote dermatologists could also present a reliable means to seek specialist input. Pathways could be established to enable the use of teledermatology.

In our analysis, there were no volunteer doctors at the stepdown facilities studied. Community-based volunteerism can contribute an important service and provide valuable health expertise to the public. ${ }^{(15)} \mathrm{A}$ visible and sustained commitment to communities may also help the medical profession to regain social trust at a time when it is increasingly under scrutiny. Finally, community volunteerism offers opportunities for physicians to better understand the social context of health and to address social determinants of health either through advocacy or service.

Interestingly, our study showed a significant delay of 2.03 months between recognition of skin issues and definitive diagnosis and management. Potential explanations include a relative scarcity of medical doctors, the logistics of transferring a patient to an external centre for review, or perhaps the persistence of nursing staff in initiating treatment and not escalating the case to receive medical attention. The reasonable time frame from recognition to definitive diagnosis and management would vary depending on the type of skin condition. Dermatological emergencies such as drug eruptions, including Stevens-Johnson syndrome and toxic epidermal necrolysis, should be diagnosed and managed within days, as these conditions are potentially fatal and can involve other organ systems. Skin infections such as scabies and fungal skin infections should be diagnosed within days to weeks due to their tendency to spread extensively on the patient's skin and to infect other patients. Immunobullous conditions such as bullous pemphigoid likewise require diagnosis within days to weeks, as they can be very symptomatic and the resultant breach in skin barrier could lead to fluid loss and infective complications; this is especially pertinent to the elderly population, which is predisposed to these conditions. Cutaneous malignancies require diagnosis early within weeks so that appropriate workup and treatment can be instituted and to prevent progression. Conversely, the time frame from recognition to diagnosis and management of chronic inflammatory skin conditions including eczema and psoriasis may be longer, such that a delay of 1-2 months is reasonable; however, this also depends on the severity of the skin condition. A delay of definitive diagnosis and management has implications. Firstly, potentially morbid skin conditions such as drug reactions or blistering diseases could remain undiagnosed and deteriorate. Secondly, inappropriate treatment could be instituted. For example, tinea pedis could be treated as endogenous eczema and progress to tinea corporis or onychomycosis. ${ }^{(16)}$ Furthermore, the usage of combination creams containing potent topical steroids and antibiotics in the face of diagnostic uncertainty unnecessarily exposes residents to side effects such as skin atrophy and antibiotic resistance.

This study was limited by the small number of participating nursing homes and their location in the west of Singapore, indicating possible selection bias. The referral centres and surrounding GP clinics that are available for consultation may be different in other parts of the nation. Furthermore, identification of dermatological diagnoses relied solely on a review of patients' medical documentation instead of a physical review by the investigators. Patients in step-down facilities were not reviewed by dermatologists, and hence no skin examinations were done to verify the diagnoses. A dermatologist's physical review of the patients could potentially have increased the diagnosis of incident skin conditions such as xerosis ${ }^{(17)}$ or other clinically significant dermatoses. With this preliminary data, further clarity on the prevalence of skin disease at step-down facilities would be provided by physical dermatological screening examinations. Our study highlights the need for comprehensive and cost-effective community dermatological care in step-down facilities. Besides improving dermatological know-how in primary care physicians and nursing staff, we hope that our findings also encourage other strategies such as volunteerism among physicians, deploying dermatologists to the community and enabling teledermatology between step-down facilities and dermatologists. The overall aim would be to prevent unnecessary clinic visits for residents and increase skin health. Future research could aim to evaluate differences in outcomes between primary and specialist care, and between various skin disorders affecting the elderly.

\section{REFERENCES}

1. Department of Statistics Singapore. Population Trends. Available at: https:// www.singstat.gov.sg/publications/population/population-trends. Accessed November 23, 2020.

2. Harrison MB, Graham ID, Lorimer K, et al. Nurse clinic versus home delivery of evidence-based community leg ulcer care: a randomized health services trial. BMC Health Serv Res 2008; 8:243

3. Jindal R, Jain A, Roy S, Rawat SD, Bhardwaj N. Skin disorders among geriatric population at a tertiary care center in Uttarakhand. J Clin Diagn Res 2016; 10:WC06-08.

4. Deo MS, Kerse N, Vandal AC, Jarrett P. Dermatological disease in the older age group: a cross-sectional study in aged care facilities. BMJ Open 2015; 5:e009941.

5. Hay RJ, Fuller LC. Global burden of skin disease in the elderly: a grand challenge to skin health. G Ital Dermatol Venereol 2015; 150:693-8.

6. Hahnel E, Lichterfeld A, Blume-Peytavi U, Kottner J. The epidemiology of skin conditions in the aged: a systematic review. J Tissue Viability 2017; 26:20-8.

7. Hay RJ, Johns NE, Williams HC, et al. The global burden of skin disease in 2010: an analysis of the prevalence and impact of skin conditions. J Invest Dermatol $2014 ; 134: 1527-34$.

8. Shah M, Coates M. An assessment of the quality of life in older patients with skin disease. Br J Dermatol 2006; 154:150-3.

9. Kim EK, Kim HO, Park YM, et al. Prevalence and risk factors of depression in geriatric patients with dermatological diseases. Ann Dermatol 2013; 25:278-84.

10. Hahnel E, Blume-Peytavi U, Trojahn C, et al. Prevalence and associated factors of skin diseases in aged nursing home residents: a multicentre prevalence study. BMJ Open 2017; 7:e018283.

11. Hahnel E, Blume-Peytavi U, Trojahn C, Kottner J. Associations between skin barrier characteristics, skin conditions and health of aged nursing home residents: a multi-center prevalence and correlational study. BMC Geriatr 2017; 17:263.

12. Nakagami G, Takehara K, Kanazawa T, et al. The prevalence of skin eruptions and mycoses of the buttocks and feet in aged care facility residents: a crosssectional study. Arch Gerontol Geriatr 2014; 58:201-4

13. Ilkit M, Durdu M. Tinea pedis: the etiology and global epidemiology of a common fungal infection. Crit Rev Microbiol 2015; 41:374-88.

14. Ministry of Health Singapore. Disease Burden. Available at: https://www.moh. gov.sg/resources-statistics/singapore-health-facts/disease-burden. Accessed August 1, 2018.

15. Grande D, Armstrong K. Community volunteerism of US physicians. J Gen Intern Med 2008; 23:1987-91.

16. Ilkit M, Durdu M. Tinea pedis: the etiology and global epidemiology of a common fungal infection. Crit Rev Microbiol 2015; 41:37.

17. Mekić S, Jacobs LC, Gunn DA, et al. Prevalence and determinants for xerosis cutis in the middle-aged and elderly population: a cross-sectional study. J Am Acad Dermatol 2019; 81:963-9.e2. [Epub ahead of print] 